\title{
Radion tunneling in modified theories of gravity
}

\author{
Tanmoy Paul ${ }^{\mathrm{a}}$, Soumitra SenGupta ${ }^{\mathrm{b}}$ \\ Department of Theoretical Physics, Indian Association for the Cultivation of Science, 2A \& 2B Raja S.C. Mullick Road, Kolkata 700 032, India
}

Received: 8 March 2018 / Accepted: 18 April 2018 / Published online: 26 April 2018

(C) The Author(s) 2018

\begin{abstract}
We consider a five dimensional warped spacetime where the bulk geometry is governed by higher curvature $F(R)$ gravity. In this model, we determine the modulus potential originating from the scalar degree of freedom of higher curvature gravity. In the presence of this potential, we investigate the possibility of modulus (radion) tunneling leading to an instability in the brane configuration. Our results reveal that the parametric regions where the tunneling probability is highly suppressed, corresponds to the parametric values required to resolve the gauge hierarchy problem.
\end{abstract}

\section{Introduction}

Over the last two decades models with extra spatial dimensions [1-13] have been increasingly playing a central role in search for physics beyond standard model of elementary particle $[14,15]$ and Cosmology $[16,17]$. Such higher dimensional scenarios occur naturally in string theory and also are viable candidates to resolve the well known gauge hierarchy problem. Depending on different possible compactification schemes for the extra dimensions, a large number of models have been constructed. In all these models, our visible universe is identified as a 3-brane embedded in a higher dimensional spacetime and is described through a low energy effective theory on the brane carrying the signatures of extra dimensions [18-20].

Among various extra dimensional models proposed over last several years, warped extra dimensional model pioneered by Randall and Sundrum (RS) [6] earned a special attention since it resolves the gauge hierarchy problem without introducing any intermediate scale (between Planck and TeV) in the theory. Subsequently different variants of warped geometry model were extensively studied in [17,21-30]. A generic feature of many of these models is that the bulk spacetime is

\footnotetext{
a e-mail: pul.tnmy9@gmail.com

b e-mail: tpssg@iacs.res.in
}

endowed with high curvature scale $\sim 4$ dimensional Planck scale.

It is well known that Einstein-Hilbert action can be generalized by adding higher order curvature terms which naturally arise from diffeomorphism property of the action. Such terms also have their origin in String theory from quantum corrections. In this context $F(R)$ [31-43], Gauss-Bonnet (GB) [4446] or more generally Lanczos-Lovelock gravity are some of the candidates in higher curvature gravitational theory.

In general the higher curvature terms are suppressed with respect to Einstein-Hilbert term by Planck scale. Hence in low curvature regime, their contributions are negligible. However higher curvature terms become extremely relevant in a region with large curvature. Thus for bulk geometry where the curvature is of the order of Planck scale, the higher curvature terms should play a crucial role. Motivated by this idea, in the present work, we consider a generalized warped geometry model by replacing Einstein-Hilbert bulk gravity action with a higher curvature $F(R)$ gravitational theory [41,42,47-53].

One of the crucial aspects of higher dimensional two brane models is to stabilize the interbrane separation (also known as modulus or radion). For this purpose, one needs to generate a suitable radion potential with a stable minimum [21-23]. The presence of such minimum guarantees the stability of the modulus field. In Goldberger-Wise stabilization mechanism [21,22], an external bulk scalar field was invoked to create such a stable radion potential. However, when the bulk is endowed with higher curvature $F(R)$ gravity, then apart from the metric there is an additional scalar degree of freedom originating from higher derivative terms of the metric. It has been shown that such a scalar degree of freedom can play the role of the stabilizing field for appropriate choices of the underlying $F(R)$ model $[26,27]$.

It is important to analyze the exact nature of the resulting radion potential to explore whether there exists a metastable minimum for the radion from which it can tunnel and leads to an instability of the braneworld [54-59]. In this paper, 
we aim to determine the radion tunneling in the presence of higher curvature gravity in the bulk.

Our paper is organized as follows: following section is devoted to brief review of the conformal relationship between $F(R)$ and scalar-tensor (ST) theory. In Sect. 3, we extend our analysis of Sect. 2 for the specific $F(R)$ model considered in this work. Section 4 extensively describes the tunneling probability for the dual ST model while Sect. 5 addresses these for the original $F(R)$ model. After discussing the equivalence, the paper ends with some concluding remarks in Sect. 6.

\section{Transformation of a $F(R)$ theory to scalar-tensor theory}

In this section, we briefly describe how a higher curvature $\mathrm{F}(\mathrm{R})$ gravity model in five dimensional scenario can be recast into Einstein gravity with a scalar field. The $F(R)$ action is expressed as,

$S=\frac{1}{2 \kappa^{2}} \int d^{4} x d \phi \sqrt{G} F(R)$

where $x^{\mu}=\left(x^{0}, x^{1}, x^{2}, x^{3}\right)$ are usual four dimensional coordinate and $\phi$ is the extra dimensional spatial angular coordinate. $R$ is the five dimensional Ricci curvature and $G$ is the determinant of the metric. Moreover $\frac{1}{2 \kappa^{2}}$ is taken as $2 M^{3}$ where $M$ is the five dimensional Planck scale. Introducing an auxiliary field $A(x, \phi)$, the action (in Eq. (1)) can be equivalently written as,

$S=\frac{1}{2 \kappa^{2}} \int d^{4} x d \phi \sqrt{G}\left[F^{\prime}(A)(R-A)+F(A)\right]$.

By the variation of the auxiliary field $A(x, \phi)$, one easily obtains $A=R$. Plugging back this solution $A=R$ into action (2), initial action (1) can be reproduced. At this stage, one may perform a conformal transformation of the metric as

$G_{M N}(x, \phi) \rightarrow \tilde{G}_{M N}=e^{\sigma(x, \phi)} G_{M N}(x, \phi)$.

$M, N$ run form 0 to $5 . \sigma(x, \phi)$ is conformal factor and related to the auxiliary field as $\sigma=(2 / 3) \ln F^{\prime}(A)$. Using this relation between $\sigma(x, \phi)$ and $A(x, \phi)$, one lands up with the following scalar-tensor action

$$
\begin{aligned}
S= & \frac{1}{2 \kappa^{2}} \int d^{4} x d \phi \sqrt{\tilde{G}}\left[\tilde{R}+3 \tilde{G}^{M N} \partial_{M} \sigma \partial_{N} \sigma\right. \\
& \left.+4 \tilde{G}^{M N} \partial_{M} \partial_{N} \sigma-\left(\frac{A}{F^{\prime}(A)^{2 / 3}}-\frac{F(A)}{F^{\prime}(A)^{5 / 3}}\right)\right],
\end{aligned}
$$

where $\tilde{R}$ is the Ricci scalar formed by $\tilde{G}_{M N} \cdot \sigma(x, \phi)$ is the scalar field emerged from higher curvature degrees of freedom. Clearly kinetic part of $\sigma(x, \phi)$ is non canonical. In order to make the scalar field canonical, transform $\sigma \rightarrow$ $\Psi(x, \phi)=\sqrt{3} \frac{\sigma(x, \phi)}{\kappa}$. In terms of $\Psi(x, \phi)$, the above action takes the form,

$$
\begin{aligned}
S= & \int d^{4} x d \phi \sqrt{\tilde{G}}\left[\frac{\tilde{R}}{2 \kappa^{2}}+\frac{1}{2} \tilde{G}^{M N} \partial_{M} \Psi \partial_{N} \Psi\right. \\
& \left.+\frac{2}{\sqrt{3} \kappa} \tilde{G}^{M N} \partial_{M} \partial_{N} \Psi-U(\Psi)\right],
\end{aligned}
$$

where $U(\Phi)=\frac{1}{2 \kappa^{2}}\left[\frac{A}{F^{\prime}(A)^{2 / 3}}-\frac{F(A)}{F^{\prime}(A)^{5 / 3}}\right]$ is the scalar field potential which depends on the form of $F(R)$. Thus the action of $F(R)$ gravity in five dimension can be transformed into the action of a scalar-tensor theory by a conformal transformation of the metric.

\section{Warped spacetime in $F(R)$ model and corresponding scalar-tensor theory}

In the present paper, we consider a five dimensional spacetime with two 3-brane scenario in $\mathrm{F}(\mathrm{R})$ model. The form of $F(R)$ is taken as $F(R)=R+\alpha R^{n}$ where $n$ takes only positive values, $\alpha$ is a constant and has the mass dimension $[2-2 n]$. Considering $\phi$ as the extra dimensional angular coordinate, two branes are located at $\phi=0$ (hidden brane) and at $\phi=\pi$ (visible brane) respectively while the latter one is identified with the visible universe. Moreover the spacetime is $S^{1} / Z_{2}$ orbifolded along the coordinate $\phi$. The action for this model is:

$$
\begin{aligned}
S= & \int d^{4} x d \phi \sqrt{G}\left[\frac{1}{2 \kappa^{2}}\left(R+\alpha R^{n}\right)\right. \\
& \left.+\frac{1}{r_{c}}\left(V_{h}+\frac{Q_{h}}{\kappa^{2}}\right) \delta(\phi)+\frac{1}{r_{c}}\left(V_{v}+\frac{Q_{v}}{\kappa^{2}}\right) \delta(\phi-\pi)\right]
\end{aligned}
$$

where $V_{h}, V_{v}$ are the brane tensions on hidden, visible brane respectively. We also include Gibbons-Hawking boundary terms on the branes, symbolized by $Q_{h}$ and $Q_{v}$ in the above action (i.e $Q_{h}, Q_{v}$ are the trace of extrinsic curvatures on hidden, visible brane respectively).

This higher curvature $F(R)$ model (in Eq. (5)) can be transformed into a scalar-tensor theory by using the technique discussed in the previous section. Performing a conformal transformation of the metric as

$$
G_{M N}(x, \phi) \rightarrow \tilde{G}_{M N}=\exp \left(\frac{1}{\sqrt{3}} \kappa \Psi(x, \phi)\right) G_{M N}(x, \phi)
$$

the above action (in Eq. (5)) can be expressed as a scalartensor theory with the action given by: 


$$
\begin{aligned}
S= & \int d^{4} x d \phi \sqrt{\tilde{G}}\left[\frac{\tilde{R}}{2 \kappa^{2}}+\frac{1}{2} \tilde{G}^{M N} \partial_{M} \Psi \partial_{N} \Psi-U(\Psi)\right. \\
& +\Lambda+\frac{2}{\sqrt{3} \kappa} \tilde{G}^{M N} \partial_{M} \partial_{N} \Psi+\frac{1}{r_{c}} e^{-\frac{5}{2 \sqrt{3}} \kappa \Psi}\left(V_{h}+\frac{Q_{h}}{\kappa^{2}}\right) \delta(\phi) \\
& \left.+\frac{1}{r_{c}} e^{-\frac{5}{2 \sqrt{3}} \kappa \Psi}\left(V_{v}+\frac{Q_{v}}{\kappa^{2}}\right) \delta(\phi-\pi)\right],
\end{aligned}
$$

where $\Lambda$ is chosen to be negative and the quantities in tilde are reserved for ST theory. $\tilde{R}$ is the Ricci curvature formed by the transformed metric $\tilde{G}_{M N} . \Psi(x, \phi)$ is the scalar field corresponds to higher curvature degree of freedom and $U(\Psi)$ is the scalar potential which for this specific form of $F(R)$ has the form,

$U(\Psi)=\Lambda+\frac{a}{\kappa^{2}} e^{b \kappa \Psi}$,

where $a$ (mass dimension [2]) and $b$ (dimensionless) are related to the parameters $\alpha$ and $n$ by the following expressions:

$$
\begin{aligned}
& \alpha=\left(\frac{2 \sqrt{3} a^{2} \kappa^{8 / 3}}{4 b+10}\right)\left[\frac{\sqrt{3} a \kappa^{8 / 3}}{4 b^{2}}\right]^{\frac{3}{2+2 \sqrt{3} b}} \\
& n=\frac{5+2 \sqrt{3} b}{2+2 \sqrt{3} b} .
\end{aligned}
$$

Considering that the scalar field depends on extra dimensional coordinate only (see Eq. (14)), the total derivative term can be integrated once leading to the final form of the action as follows:

$$
\begin{aligned}
S= & \int d^{4} x d \phi \sqrt{\tilde{G}}\left[\frac{\tilde{R}}{2 \kappa^{2}}+\frac{1}{2} \tilde{G}^{M N} \partial_{M} \Psi \partial_{N} \Psi-U(\Psi)\right. \\
& +\Lambda+\left(\frac{1}{r_{c}} e^{-\frac{5}{2 \sqrt{3}} \kappa \Psi}\left(V_{h}+\frac{Q_{h}}{\kappa^{2}}\right)+\frac{2}{\sqrt{3} \kappa} \tilde{G}^{\phi \phi} \frac{\partial \Psi}{\partial \phi}\right) \delta(\phi) \\
& \left.+\left(\frac{1}{r_{c}} e^{-\frac{5}{2 \sqrt{3}} \kappa \Psi}\left(V_{v}+\frac{Q_{v}}{\kappa^{2}}\right)+\frac{2}{\sqrt{3} \kappa} \tilde{G}^{\phi \phi} \frac{\partial \Psi}{\partial \phi}\right) \delta(\phi-\pi)\right] .
\end{aligned}
$$

\section{Radion potential and tunneling probability in scalar-tensor (ST) theory}

In order to generate radion potential in ST theory, here we adopt the GW mechanism [22] which requires a scalar field in the bulk. For the case of ST theory presented in Eq. (10), $\Psi$ can act as a bulk scalar field. Considering a negligible backreaction of the scalar field $(\Psi)$ on the background spacetime, the solution of metric $\tilde{G}_{M N}$ is exactly same as well known RS model i.e.

$d \tilde{s}^{2}=e^{-2 k r_{c}|\phi|} \eta_{\mu \nu} d x^{\mu} d x^{\nu}-r_{c}^{2} d \phi^{2}$

where $k=\sqrt{\frac{-\Lambda}{24 M^{3}}}$. Using these metric and explicit form of $U(\Psi)$, we obtain the scalar field equation of motion in the bulk as follows, $\frac{1}{r_{c}^{2}} \frac{\partial^{2} \Psi}{\partial \phi^{2}}-4 \frac{k}{r_{c}} \frac{\partial \Psi}{\partial \phi}+\frac{a b}{\kappa} e^{b \kappa \Psi}=0$.

To derive the above equation of motion, $\Psi$ is taken as function of extra dimensional coordinate only. Considering the variation of $\Psi(\phi)$ is small in the bulk [21,22], Eq. (12) turns out to be,

$$
-4 \frac{k}{r_{c}} \frac{\partial \Psi}{\partial \phi}+\frac{a b}{\kappa} e^{b \kappa \Psi}=0 .
$$

With a non zero value of $\Psi$ on the branes, above equation has the following solution:

$e^{b \kappa \Psi(\phi)}=\frac{4 k}{a b^{2}}\left[\frac{1}{y_{0}-r_{c} \phi}\right]$

where $y_{0}=\frac{4 k}{a b^{2}} e^{-b \kappa v_{h}}$ and $v_{h}$ is the value of the bulk scalar field on the hidden brane $(\phi=0)$.

Using the solution of metric (see Eq. (11)), we obtain the extrinsic curvature of $\phi=$ constant hypersurface as follows:

$Q_{\mu \nu}=k e^{-2 k r_{c} \phi} \eta_{\mu \nu}$

and

$Q=Q_{\mu \nu} e^{2 k r_{c} \phi} \eta^{\mu \nu}=4 k$

The above expression of $Q$ (trace of the extrinsic curvature) leads to the boundary term of the action as,

$$
\begin{aligned}
S_{b}= & \int d^{4} x\left[\sqrt{-g_{h}}\left(e^{-\frac{5}{2 \sqrt{3}} \kappa v_{h}}\left(V_{h}+\frac{4 k}{\kappa^{2}}\right)-\frac{a b}{2 \sqrt{3} k \kappa^{2}} e^{b \kappa v_{h}}\right)\right. \\
& \left.+\sqrt{-g_{v}}\left(e^{-\frac{5}{2 \sqrt{3}} \kappa v_{v}}\left(V_{v}+\frac{4 k}{\kappa^{2}}\right)-\frac{a b}{2 \sqrt{3} k \kappa^{2}} e^{b \kappa v_{v}}\right)\right] \\
= & \int d^{4} x\left[\sqrt{-g_{h}} V_{h}^{e f f}+\sqrt{-g_{v}} V_{v}^{e f f}\right]
\end{aligned}
$$

where we use the explicit solution of $\Psi(\phi)$ (see Eq. (14)) with $v_{h}=\Psi(0), v_{v}=\Psi(\pi)$. Further

$V_{h}^{e f f}=e^{-\frac{5}{2 \sqrt{3}} \kappa v_{h}}\left(V_{h}+\frac{4 k}{\kappa^{2}}\right)-\frac{a b}{2 \sqrt{3} k \kappa^{2}} e^{b \kappa v_{h}}$

and

$V_{v}^{e f f}=e^{-\frac{5}{2 \sqrt{3}} \kappa v_{v}}\left(V_{v}+\frac{4 k}{\kappa^{2}}\right)-\frac{a b}{2 \sqrt{3} k \kappa^{2}} e^{b \kappa v_{v}}$,

with $g_{h}, g_{v}$ are the determinants of the induced metric on hidden, visible brane respectively. It may be observed that the boundary terms emerging from the total derivative of $\Psi$ and the Gibbons-Hawking terms modify the brane tensions of the respective branes to produce the effective brane tensions as $V_{h}^{\text {eff }}$ and $V_{v}^{\text {eff }}$.

Plugging back the solution of $\Psi(\phi)$ (Eq. (14)) into scalar field action and integrating over $\phi$ yields an effective modulus potential having the form as, 


$$
\begin{aligned}
V_{e f f}\left(r_{c}\right)= & \frac{1}{b^{2} \kappa^{2}}\left[\frac{e^{-4 k r_{c} \pi}}{\pi r_{c}-y_{0}}+\frac{1}{y_{0}}\right]-\frac{\Lambda}{2 k}\left[1-e^{-4 k r_{c} \pi}\right] \\
& -\frac{4 k}{b^{2} \kappa^{2}} e^{-4 k y_{0}}\left(E i\left[4 k\left(y_{0}-\pi r_{c}\right)\right]-E i\left[4 k y_{0}\right]\right),
\end{aligned}
$$

where 'Ei' denotes the exponential integral function.

It may be observed that the scalar field degrees of freedom is related to the curvature as,

$\Psi(\phi)=\frac{2}{\sqrt{3} \kappa} \ln \left[1+n \alpha R^{n-1}\right]$

From the above expression, we can relate the boundary values of the scalar field (i.e $\Psi(0)=v_{h}$ ) with the Ricci scalar as,

$v_{h}=\frac{2}{\sqrt{3} \kappa} \ln \left[1+n \alpha R^{n-1}(0)\right]$,

where $R(0)$ is the value of the curvature on Planck brane. Thus the parameters that are used in the scalar-tensor theory are actually related to the parameters of the original $F(R)$ theory.

Furthermore the various components of stress tensor of the scalar field $\Psi$ can be written as,

$T_{\mu \nu}(\Psi)=-\frac{1}{2} \eta_{\mu \nu} e^{-2 k r_{c}|\phi|}\left[|\Lambda|-\frac{1}{2 \kappa^{2} b^{2}} \frac{1}{\left(r_{c} \phi-y_{0}\right)^{2}}\right]$

and

$T_{\phi \phi}(\Psi)=\frac{1}{2} r_{c}^{2}\left[|\Lambda|-\frac{3}{2 \kappa^{2} b^{2}} \frac{1}{\left(r_{c} \phi-y_{0}\right)^{2}}\right]$,

where we use the solution of $\Psi(\phi)$ obtained in Eq. (14). These above expressions of $T_{M N}(\Psi)$ lead to the ratio of corresponding component of stress tensor between bulk scalar field and bulk cosmological constant as,

$\left(\frac{T_{\mu \nu}(\Psi)}{T_{\mu \nu}(\Lambda)}\right)_{\max }=1-\frac{a^{2} b^{2}}{32 \kappa^{2} k^{2}|\Lambda|} e^{2 b \kappa v_{h}}$

and

$\left(\frac{T_{\phi \phi}(\Psi)}{T_{\phi \phi}(\Lambda)}\right)_{\max }=1-\frac{3 a^{2} b^{2}}{32 \kappa^{2} k^{2}|\Lambda|} e^{2 b \kappa v_{h}}$,

where $T_{\phi \phi}(\Lambda)$ and $T_{\mu \nu}(\Lambda)$ are different components of stress tensor for the bulk cosmological constant. It may be observed that for $e^{b \kappa v_{h}}<\frac{\kappa k \sqrt{|\Lambda|}}{a b}$, the stress tensor for the scalar field $(\Psi)$ is less than that for the bulk cosmological constant $(\Lambda)$ for entire range of extra dimensional coordinate (i.e $0<\phi<\pi$ ). This condition allows us to neglect the back-reaction of the scalar field in comparison to bulk cosmological constant.

To introduce the radion field we replace $r_{c} \rightarrow \tilde{T}(t)$ [22], where $\tilde{T}(t)$ is the fluctuation of the modulus around its vev and is known as radion field. Here, for simplicity we assume
[22] that this new field depends only on $t$. The corresponding metric ansatz is,

$d \tilde{s}^{2}=e^{-2 k \tilde{T}(t)|\phi|} \eta_{\mu \nu} d x^{\mu} d x^{\nu}-\tilde{T}^{2}(t)^{2} d \phi^{2}$.

Recall that the quantities in tilde are reserved for ST theory. As mentioned earlier, the bulk scalar field $\Psi$ fulfills the requirement for generating the radion potential.

With the metric in Eq. (23), the five dimensional EinsteinHilbert part of the action yields the kinetic part of the radion field in the four dimensional effective action as [22],

$S_{k i n}[\tilde{T}]=\frac{12 M^{3}}{k} \int d^{4} x \partial_{\mu}\left(e^{-k \pi \tilde{T}(t)}\right) \partial^{\mu}\left(e^{-k \pi \tilde{T}(t)}\right)$.

As we see that $\tilde{T}(t)$ is not canonical and thus we redefine the field by the following transformation,

$\tilde{T}(t) \longrightarrow \tilde{T}_{c a n}(t)=\sqrt{\frac{24 M^{3}}{k}} e^{-k \pi \tilde{T}(t)}$

Correspondingly the radion potential is obtained from Eq. (20) by replacing $r_{c}$ by $\tilde{T}(t)$ i.e.

$$
\begin{aligned}
V_{S T}(\tilde{T})= & \frac{1}{b^{2} \kappa^{2}}\left[\frac{e^{-4 k \pi \tilde{T}(t)}}{\pi \tilde{T}(t)-y_{0}}+\frac{1}{y_{0}}\right]-\frac{\Lambda}{2 k}\left[1-e^{-4 k \pi \tilde{T}}\right] \\
& -\frac{4 k}{b^{2} \kappa^{2}} e^{-4 k y_{0}}\left(\operatorname{Ei}\left[4 k\left(y_{0}-\pi \tilde{T}\right)\right]-E i\left[4 k y_{0}\right]\right) .
\end{aligned}
$$

In terms of $\tilde{T}_{c a n}$, the Lagrangian of radion field becomes

$L\left[\tilde{T}_{c a n}\right]=\left[\frac{1}{2} \tilde{\dot{T}}_{c a n}^{2}-V_{S T}\left(\tilde{T}_{c a n}\right)\right]$

which is the same Lagrangian for a particle moving in a potential $V_{S T}$.

The potential $V_{S T}$ has a minimum at

$$
<\pi \tilde{T}>_{+}=<\pi r_{c}>_{+}=y_{0}-\frac{2 k}{b^{2} \kappa^{2} \Lambda}\left[\sqrt{1-\frac{b^{2} \kappa^{2} \Lambda}{8 k^{2}}}+1\right]
$$

and a maxima at

$$
\begin{aligned}
<\pi \tilde{T}>_{-} & =<\pi r_{c}>_{-} \\
& =y_{0}-\frac{2 k}{b^{2} \kappa^{2} \Lambda}\left[\sqrt{1-\frac{b^{2} \kappa^{2} \Lambda}{8 k^{2}}}-1\right] .
\end{aligned}
$$

respectively. Recall $y_{0}=\frac{4 k}{a b^{2}} e^{-b \kappa v_{h}}, a$ and $b$ are given by Eq. (9). Moreover, Eq. (25) clearly indicates that $V_{S T}(\tilde{T})$ becomes zero at $\tilde{T}=0$ and reaches a constant value ( $=$ $\left.\frac{1}{b^{2} \kappa^{2} y_{0}}-\frac{\Lambda}{2 k}+\frac{4 k}{b^{2} \kappa^{2}} e^{-4 k y_{0}} E i\left[4 k y_{0}\right]\right)$ asymptotically at large $\tilde{T}$. In Fig. 1, we give the plot between $V_{S T}(\tilde{T})$ versus $\tilde{T}(t)$. 


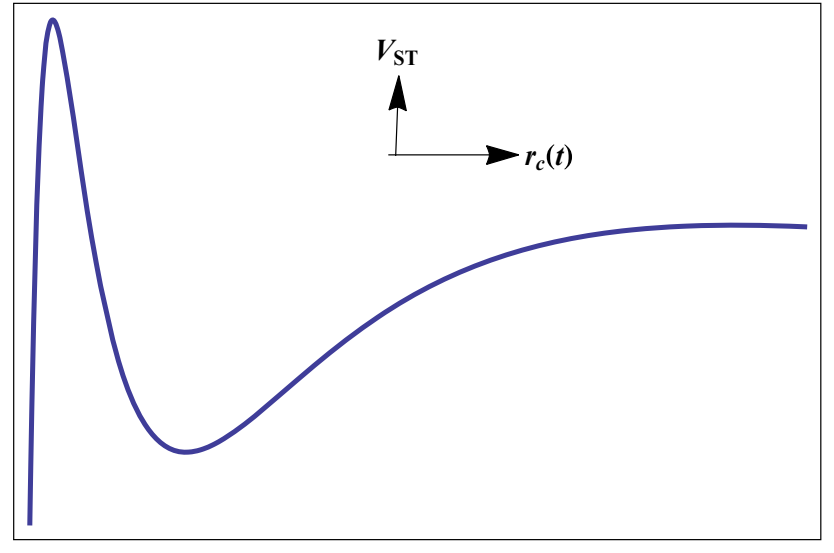

Fig. $1 V_{S T}$ vs $r_{c}(t)(=\tilde{T}(t))$ for $a=1, b=\frac{\sqrt{2}}{3}, k=M=1, \Lambda=$ $-1, \kappa v_{h}=0.01$

Consequently, using the form of radion potential in Eq. (25) with the transformation given in Eq. (24), one arrives at the following mass squared of radion field in scalar-tensor theory given as,

$$
\begin{aligned}
\tilde{m}_{r a d}^{2}(S T)= & e^{-2 k \pi<r_{c}>+\left(\frac{b^{2} \kappa^{2} \Lambda^{2}}{12 M^{3} k^{2}}\right)} \\
& \times\left[\frac{\sqrt{1-\frac{b^{2} \kappa^{2} \Lambda}{8 k^{2}}}}{\left(1+\sqrt{1-\frac{b^{2} \kappa^{2} \Lambda}{8 k^{2}}}\right)^{2}}\right] .
\end{aligned}
$$

According to Goldberger-Wise (GW) stabilization mechanism [21,22], the modulus is stabilized at that separation for which the effective radion potential becomes minimum. Therefore, in the present context, the stable value of interbrane separation is given by $r_{c}=<r_{c}>_{+}$, which is determined in Eq. (26). But due to quantum fluctuation, the radion field has a non zero probability to tunnel from $r_{c}=<r_{c}>_{+}$ to $r_{c}=0$, which in turn makes the brane configuration unstable. So it is worthwhile to calculate the quantum tunneling for radion field from $r_{c}=<r_{c}>_{+}$to $r_{c}=0$. In order to do so, the radion potential is approximately considered as a rectangle barrier having width $(w)=\left\langle r_{c}\right\rangle_{+}$and height $(h)$ $\left.=V_{e f f}\left(<r_{c}\right\rangle_{-}\right)$respectively. For such a potential barrier, the tunneling probability $\left(P_{S T}\right)$ is given by,

$$
\begin{aligned}
\frac{1}{P_{S T}} & =1+\left(\frac{V_{S T}\left(<r_{c}>_{-}\right)}{\Delta V_{S T}}\right) \sinh ^{2}\left[\pi \sqrt{\frac{2 \tilde{m}_{r a d} \Delta V_{S T}}{M^{3}}} *\right. \\
& \left.<r_{c}>_{+} e^{-\frac{3}{2} k \pi<r_{c}>_{+}}\right]
\end{aligned}
$$

where $\tilde{m}_{\text {rad }}$ is the mass of radion field, determined in Eq. (28) and $\Delta V_{S T}=V_{S T}\left(<r_{c}>_{-}\right)-V_{S T}\left(<r_{c}>_{+}\right)$, which can be easily calculated from the expression of radion potential.

Obviously, $P_{S T}$ depends on the parameters $a$ and $b$. For $a=1$ (in Planckian unit), we give the plot between $P_{S T}$ versus $b$ (see Fig. 2):

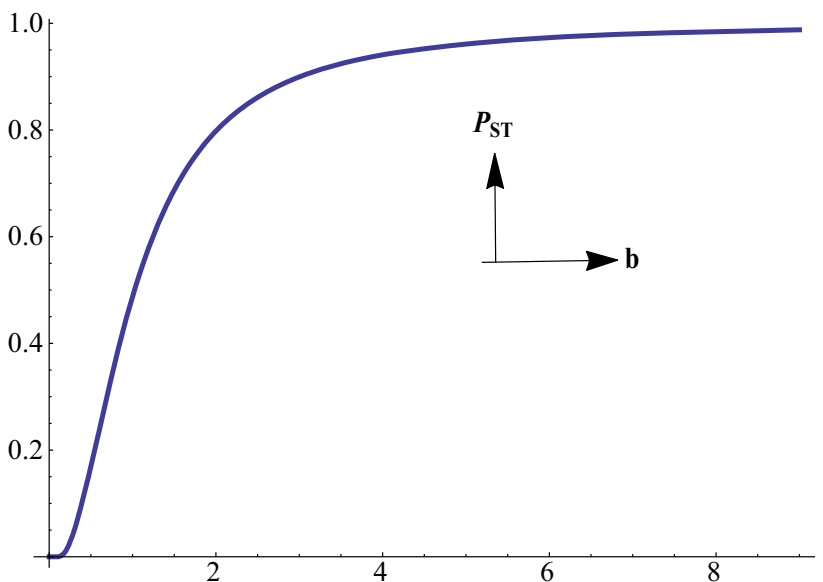

Fig. $2 P_{S T}$ vs $b$ for $a=1, k=M=1, \Lambda=-1, \kappa v_{h}=0.01$

Figure 2 clearly depicts that the tunneling probability increases with the parameter $b$ and asymptotically reaches to unity at large $b$. It is expected, because with increasing value of $b$, the height as well as the width ( both are $\propto \frac{1}{b^{2}}$ ) of the potential barrier decreases and as a consequence, $P_{S T}$ increases. Moreover, $P_{S T}$ becomes zero as $b$ tends to zero, because for $b \rightarrow 0$, the height of the potential barrier goes to infinite and as a result, $P_{S T}=0$. This character of global minimum (as $b$ tends to zero) actually overlaps with the Goldberger-Wise result [21,22].

However, resolution of the gauge hierarchy problem requires $k \pi<r_{c}>_{+}=36$, which in turn makes $b \simeq \frac{1}{3 \sqrt{3}}$ (for $a=1$ ). With these values of $a$ and $b, P_{S T}$ becomes drastically suppressed and comes as $\sim 10^{-32}$. This small value of tunneling probability guarantees the stability of the interbrane separation (and hence of the radion field) at $\left\langle r_{c}\right\rangle_{+}$. Thus it can be argued that the smallness of tunneling probability is intimately connected with the requirement of resolving the gauge hierarchy problem. Further it may be mentioned that these values of $a$ and $b$ are consistent with the condition $e^{b \kappa v_{h}}<\frac{\kappa k \sqrt{|\Lambda|}}{a b}$, necessary for neglecting the backreaction of the scalar field $\Psi$ in the background spacetime (as mentioned earlier).

Now we turn our focus on radion potential as well as on radion tunneling probability for the original $F(R)$ model (Eq. (5)).

\section{Radion potential and tunneling probability in $F(R)$ model}

Recall that the original higher curvature $F(R)$ model is described by the action given in Eq. (5). Solutions of metric $\left(G_{M N}\right)$ for this $F(R)$ model can be extracted from the solutions of corresponding scalar-tensor theory (Eqs. (11) and (14)) with the help of Eq. (6). Thus the line element (in the bulk) in $F(R)$ model turns out to be 
$d s^{2}=e^{-\frac{\kappa}{\sqrt{3}} \Psi(\phi)}\left[e^{-2 k r_{c}|\phi|} \eta_{\mu \nu} d x^{\mu} d x^{\nu}-r_{c}^{2} d \phi^{2}\right]$,

where $\Psi(\phi)$ is given in Eq. (14).

At this point, we need to verify whether the above solution of $G_{M N}$ (in Eq. (30)) satisfies the field equations of the original $F(R)$ theory. The five dimensional gravitational field equation for $F(R)$ theory is given by,

$\frac{1}{2} G_{M N} F(R)-R_{M N} F^{\prime}(R)-G_{M N} \square F^{\prime}(R)$

$+\nabla_{M} \nabla_{N} F^{\prime}(R)=0$

In the present context, we take the form of $F(R)$ as $F(R)=R+\alpha R^{n}$ and thus the above field equation is simplified to the form:

$\frac{1}{2} G_{M N} R-R_{M N}+\frac{\alpha}{2} G_{M N} R^{n}-n \alpha R^{n-1} R_{M N}$

$-n \alpha G_{M N} \square R^{n-1}+n \alpha \nabla_{M} \nabla_{N} R^{n-1} .=0$

It may be shown that the solution of $G_{M N}$ in Eq. (30) satisfies the above field equation to the leading order of $\kappa v_{h}$. It may be recalled that the equivalence of the chosen $F(R)$ model was transformed to the potential of the scalar-tensor model in the leading order of $\kappa v_{h}$. Thus it guarantees the validity of the solution of spacetime metric (i.e. $G_{M N}$ ) in the original $F(R)$ theory.

However this solution of $G_{M N}$ immediately leads to the separation between hidden $(\phi=0)$ and visible $(\phi=\pi)$ branes along the path of constant $x^{\mu}$ as follows:

$\pi d=r_{c} \int_{0}^{\pi} d \phi e^{-\frac{\kappa}{2 \sqrt{3}} \Phi(\phi)}$,

where $d$ is the interbrane separation in $F(R)$ model. Using the explicit functional form of $\Psi(\phi)$ (Eq. (14)), above equation can be integrated and simplified to the following one,

$k \pi d=k \pi r_{c}\left[1-\frac{\kappa}{2 \sqrt{3}} v_{h}+\frac{a b^{2}}{16 \sqrt{3} k} \pi r_{c}\right]$,

where the sub-leading terms of $\kappa \Psi$ are neglected. $r_{c}$ is the modulus in the corresponding ST theory and has a stable value at $\left\langle r_{c}\right\rangle_{+}$, which is shown in the previous section (see Eq. (26)). So, it can be argued that due to the stabilization of ST theory, the modulus $d$ in $F(R)$ model has also a stable value at,

$$
\begin{aligned}
k \pi<d>_{+}= & k \pi<r_{c}>_{+}\left[1-\frac{\kappa}{2 \sqrt{3}} v_{h}+\frac{1}{4 \sqrt{3}} e^{-b \kappa v_{h}}\right. \\
& \left.-\frac{a}{\left(8 \sqrt{3} \kappa^{2} \Lambda\right)}\left(1+\sqrt{1-\frac{b^{2} \kappa^{2} \Lambda}{8 k^{2}}}\right)\right] .
\end{aligned}
$$

A fluctuation of branes around the stable configuration is now considered. This fluctuation can be taken as a field
$(T(t))$ and for simplicity, this new field is assumed to be the function of $t$. The metric takes the following form,

$$
\begin{aligned}
d s^{2}= & e^{-\frac{\kappa}{\sqrt{3}} \Psi(t, \phi)}\left[e^{-2 k T(t)|\phi|} \eta_{\mu \nu} d x^{\mu} d x^{\nu}\right. \\
& \left.-T(t)^{2} d \phi^{2}\right] .
\end{aligned}
$$

From the angle of four dimensional effective theory, $T(t)$ is known as radion field. Moreover $\Psi(t, \phi)$ is obtained from Eq. (14) by replacing $r_{c}$ to $T(t)$. Plugging back the solution (see Eq. (35)) into five dimensional action and integrating over $\phi$ generates a kinetic as well as a potential part for the radion field $T(t)$. Kinetic part comes as

$S_{k i n}[T]=\frac{1}{2} f^{2} \int d^{4} x \partial_{\mu}\left(e^{-k \pi T(x)}\right) \partial^{\mu}\left(e^{-k \pi T(x)}\right)$

where the factor $f$ has the following form:

$f=\sqrt{\frac{24 M^{3}}{k}}\left[1+\left(\frac{40}{\sqrt{3}}\left(\frac{\sqrt{3} a k^{2} \kappa^{8 / 3}}{4 b^{2}}\right)^{\frac{3}{2+2 \sqrt{3} b}} \kappa v_{h}\right)^{\frac{5+2 \sqrt{3} b}{4+4 \sqrt{3} b}}\right]^{1 / 2}$.

Due to the appearance of $f, T(x)$ is not canonical and in order to make it canonical, we redefine the field as

$T(x) \longrightarrow T_{c a n}(x)=f e^{-k \pi T(x)}$.

For $a \rightarrow 0$, the action contains only the linear term in Ricci scalar and the factor $f$ goes to $\sqrt{24 M^{3} / k}$ which agrees with [22].

Finally the potential part of radion field is as follows,

$$
\begin{aligned}
V_{F(R)}\left(T_{c a n}\right)= & \frac{20}{\sqrt{3}}\left(\frac{f^{4} k^{\frac{5+2 \sqrt{3} b}{1+\sqrt{3} b}}}{M^{6}}\right)\left(\frac{\sqrt{3} a \kappa^{8 / 3}}{4 b^{2}}\right)^{\frac{3}{2+2 \sqrt{3} b}} \\
& \times\left[1+\frac{4}{\sqrt{3}}\left(\kappa v_{h}\right)^{\frac{5+2 \sqrt{3} b}{4+4 \sqrt{3} b}}\right] \\
& *\left[\frac { 1 } { b ^ { 2 } \kappa ^ { 2 } } \left(\frac{1}{y_{0}}-\frac{k T_{c a n}^{4}}{f^{4}\left(y_{0}-\ln f+\ln T_{c a n}\right)}\right.\right. \\
& \left.-\frac{b^{2} \kappa^{2} \Lambda}{2 k}\left(1-\frac{T_{c a n}^{4}}{f^{4}}\right)\right)-\frac{4 k}{b^{2} \kappa^{2}} e^{-4 k y_{0}} \\
& \left.*\left(E i\left[4\left(k y_{0}-\ln f+\ln T_{c a n}\right)\right]-E i\left[4 k y_{0}\right]\right)\right] .
\end{aligned}
$$

It may be observed that $V_{F(R)}\left(T_{c a n}\right)$ goes to zero as the parameter $a$ tends to zero. This is expected because for $a \rightarrow 0$, the action contains only the Einstein part ((recall from Eq. (9) that the higher curvature parameter $\alpha$ is proportional to $a$ )) which does not produce any potential term for the radion field [22]. Thus for five dimensional warped geometric model, the radion potential is generated from the higher order curvature term $\alpha R^{n}$. Again the Lagrangian for the canonical radion takes the following form: 
$L\left[T_{c a n}\right]=\left[\frac{1}{2} \dot{T}_{c a n}^{2}-V_{F(R)}\left(T_{c a n}\right)\right]$,

which matches with the Lagrangian for a point particle moving under a potential $V_{F(R)}$.

The radion potential in $F(R)$ model has also a minima and a maxima at $<T_{\text {can }}>_{+}$and at $<T_{\text {can }}>_{-}$respectively, where

$<T_{\text {can }}>_{+}=f e^{-k \pi<d>_{+}}$

and

$<T_{c a n}>_{-}=f e^{-k \pi<d>_{-}}$

with $<d>_{+},<d>_{-}$have the following expressions:

$$
\begin{aligned}
<d>_{ \pm} & =<r_{c}>_{ \pm}\left[1-\frac{\kappa}{2 \sqrt{3}} v_{h}+\frac{1}{4 \sqrt{3}} e^{-b \kappa v_{h}}\right. \\
& \left.\mp \frac{1}{8 \sqrt{3}} \frac{a}{\kappa^{2} \Lambda}\left(\sqrt{1-\frac{b^{2} \kappa^{2} \Lambda}{8 k^{2}}} \pm 1\right)\right]
\end{aligned}
$$

where $<r_{c}>_{ \pm}$are determined in Eq. (26) and in Eq. (27) respectively. We emphasize that due to the presence of conformal factor connecting the two theories, the value of $<d>_{ \pm}$(in $F(R)$ model) is different from $\left\langle r_{c}\right\rangle_{ \pm}$(in ST model). Finally the squared mass of radion field is as follows,

$$
\begin{aligned}
& m_{r a d}^{2}=e^{-2 k \pi<d>+}\left(\frac{5}{3 \sqrt{3}}\right)\left(\frac{\sqrt{3} a \kappa^{8 / 3}}{4 b^{2}}\right)^{\frac{3}{2+2 \sqrt{3} b}}\left(\frac{k^{\frac{7+4 \sqrt{3} b}{1+\sqrt{3} b}}}{M^{2}}\right) \\
& *\left[1+\frac{20}{\sqrt{3}}\left(\left(\frac{\sqrt{3} a k^{2} \kappa^{8 / 3}}{4 b^{2}}\right)^{\frac{3}{2+2 \sqrt{3} b}} \kappa v_{h}\right)^{\frac{5+4 \sqrt{3} b}{4+4 \sqrt{3} b}}\right] \\
& {\left[\frac{\sqrt{1-\frac{b^{2} \kappa^{2} \Lambda}{8 k^{2}}}}{\left[1+\sqrt{1-\frac{b^{2} \kappa^{2} \Lambda}{8 k^{2}}}\right]^{2}}\right]}
\end{aligned}
$$

It is evident that mass of the radion field also goes to zero as $a \rightarrow 0$ (higher curvature parameter $\alpha$ is proportional to $a$ ).

Using the form of $V_{F(R)}\left(T_{c a n}\right)$ along with the transformation Eq. (38), now we give the plot between radion potential and $T(t)$ (see Fig. 3).

Figure 3 clearly depicts that the radion potential goes to zero at $T(x)=0$ and reaches a constant value asymptotically at large value of $T(x)$. Comparing Figs. 1 and 3, it is clear that the nature of radion potential does not change in comparison to that in ST theory. However, due to the conformal factor, the extremas of the potential are shifted in $F(R)$ model, which is clear from Eq. (33).

As per GW mechanism, the radion field is stabilized at $<d>_{+}$. But as mentioned earlier, due to quantum mechanical tunneling effect, there exists a non zero tunneling probability of the radion field from $d=<d>_{+}$to $d=0$. Again considering the radion potential as a rectangle barrier having width $\left(w=<d>_{+}\right)$and height $\left(h=V_{F(R)}\left(<d_{-}>\right)\right)$, we

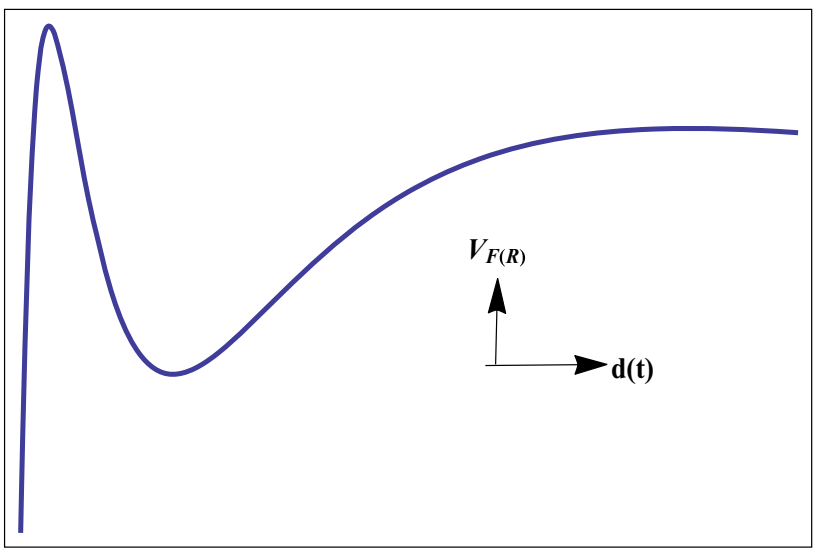

Fig. $3 V_{F(R)}$ vs $d(t)(=T(t))$ for $a=1, b=\frac{\sqrt{2}}{3}, k=M=1, \Lambda=$ $-1, \kappa v_{h}=0.01$

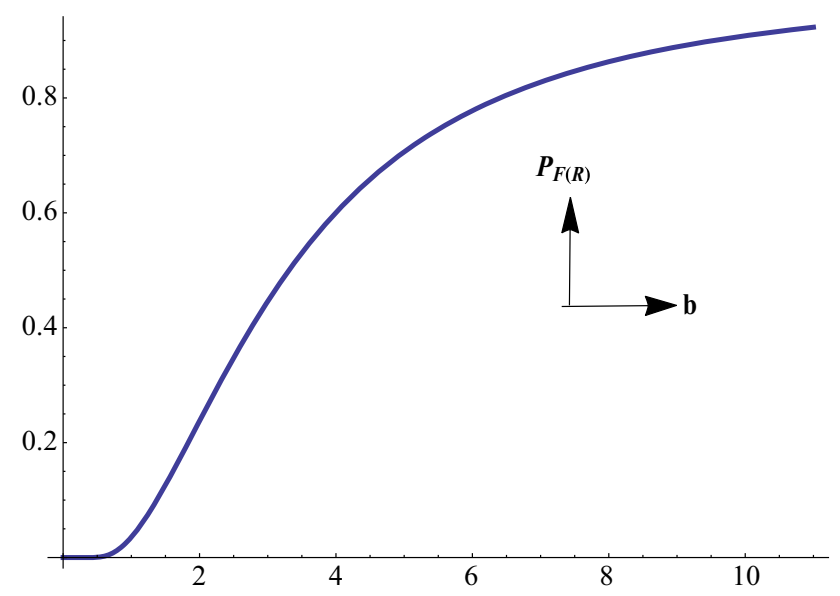

Fig. $4 P_{F(R)}$ vs $b$ for $a=1, k=M=1, \Lambda=-1, \kappa v_{h}=0.01$

calculate the tunneling probability $\left(P_{F(R)}\right)$ from $d=<d>_{+}$ to $d=0$ and is given by,

$$
\begin{aligned}
\frac{1}{P_{F(R)}} & =1+\left(\frac{V_{F(R)}\left(<d>_{-}\right)}{\Delta V_{F(R)}}\right) \sinh ^{2}\left[\pi \sqrt{\frac{2 m_{\text {rad }} \Delta V_{F(R)}}{M^{3}}} *\right. \\
& \left.<d>_{+} e^{-\frac{3}{2} k \pi<d>_{+}}\right]
\end{aligned}
$$

where $m_{\text {rad }}$ is given in Eq.(43) and $\Delta V_{F(R)}=V_{F(R)}(<$ $\left.d_{-}>\right)-V_{F(R)}\left(<d_{+}>\right)$. From Eq. (44), it is clear that $P_{F(R)}$ depends on both the parameters $a$ and $b$. Here we take $a=1$ (in Planckian unit) and give the plot demonstrating the variation of $P_{F(R)}$ with respect to $b$ (see Fig. (4)).

Figure 4 reveals that just as in ST theory, $P_{F(R)}$ (tunneling probability in $\mathrm{F}(\mathrm{R})$ model) increases with increasing value of $b$ and acquires the maximum value $(=1)$ asymptotically at large $b$. For $b \rightarrow \infty$, the higher curvature parameter $(\alpha)$ goes to zero (see Eq. (9)) and the action reduces to EinsteinHilbert action. This in turn makes the brane configuration unstable [22] and as a consequence the tunneling probability becomes unity. As the parameter $b$ decreases, the effect of 
higher curvature term starts to contribute and as a result, the modulus is stabilized at a certain separation and hence the probability for tunneling becomes less than one. Furthermore for $b \rightarrow 0$, higher curvature parameter $\alpha \rightarrow \infty$, which in turn makes the height of the radion potential barrier infinity (height $\propto \frac{1}{b^{2}}$ ) and thus the potential acquires a global minimum. As a consequence, the tunneling probability tends to zero, which is shown in Fig. 4). The character of global minimum actually mimics the result of Goldberger and Wise [21]. It is expected because for $b \rightarrow 0$, the bulk scalar potential in the present context $(U(\Psi))$ becomes quadratic (all the other terms are proportional to higher power of $b$ and can be neglected) as same as the potential considered in [21].

Finally we examine whether the solution of gauge hierarchy problem in $\mathrm{F}(\mathrm{R})$ model leads to a small value of the tunneling probability or not. We find that the resolution of gauge hierarchy problem requires $k \pi<d>_{+}=36$, which in turn makes $b=\frac{\sqrt{2}}{3}$. For this value of $b, P_{F(R)}$ is highly suppressed and takes the value of $\sim 10^{-32}$. Therefore, in original $F(R)$ theory, the requirement for solving the gauge hierarchy problem is correlated with the smallness of radion tunneling probability (a similar analysis is also obtained in ST theory as discussed in Sect. 4).

\section{Conclusion}

In this work, we consider a five dimensional compactified warped geometry model with two 3-branes embedded within the spacetime. Due to large curvature ( $\sim$ Planck scale), the bulk spacetime is governed by a higher curvature theory like $F(R)=R+\alpha R^{n}$. In this scenario, we determine the radion potential from the scalar degrees of freedom of higher curvature gravity and investigate the possibility of tunneling for the radion field. Our findings and implications are as follows:

- Due to the presence of higher curvature gravity in the bulk, a potential term for the radion field is generated, as shown in Eq. (39). This is in sharp contrast to a model with only Einstein term in the bulk where the modulus potential can not be generated without incorporating any external degrees of freedom such as a scalar field. However for the higher curvature gravity model, this additional degree of freedom originates naturally from the higher curvature term. It may also be noted that the radion potential goes to zero as the higher curvature parameter $\alpha \rightarrow 0$.

- The radion potential $\left(V_{F(R)}\right)$ has a minimum $\left(<d>_{+}\right)$ and a maximum $\left(<d>_{-}\right)$respectively where the height between minimum and maximum of the potential depends on both the parameters $\alpha$ and $n$. Moreover, $V_{F(R)}$ becomes zero at $T(x)=0(T(x)$ is the radion field) and reaches a constant value asymptotically at large $T(x)$, as depicted in Fig. 3.
- According to GW mechanism, the modulus is stabilized at $\langle d\rangle_{+}$. But due to quantum mechanical effect, there exists a possibility of tunneling for the radion field from $d=<d>_{+}$to $d=0$, which in turn makes the aforementioned brane configuration unstable. We calculate this tunneling probability $\left(P_{F(R)}\right)$ which depends on the parameters $a$ and $b$ ( $a$ and $b$ can be written in terms of $\alpha$ and $n$, see Eq. (9)). For a certain choice of $a, P_{F(R)}$ increases with increasing value of $b$, as demonstrated in Fig. 4. It may be observed that this behaviour of $P_{F(R)}$ with the parameter $b$ is expected, because the height of the potential barrier decreases as $b$ increases and as a result, $P_{F(R)}$ increases. Finally we find that the solution of gauge hierarchy problem requires $k \pi<d>_{+}=36$, which in turn highly suppresses the tunneling probability and as a consequence, $P_{F(R)}$ comes as $\sim 10^{-32}$. This small value of the tunneling probability guarantees the stability of interbrane separation at $\langle d\rangle_{+}$. Therefore, it can be argued that the smallness of tunneling probability is interrelated with the requirement of solving the gauge hierarchy problem.

Open Access This article is distributed under the terms of the Creative Commons Attribution 4.0 International License (http://creativecomm ons.org/licenses/by/4.0/), which permits unrestricted use, distribution, and reproduction in any medium, provided you give appropriate credit to the original author(s) and the source, provide a link to the Creative Commons license, and indicate if changes were made. Funded by SCOAP ${ }^{3}$.

\section{References}

1. N. Arkani-Hamed, S. Dimopoulos, G. Dvali, Phys. Lett. B 429, 263 (1998)

2. N. Arkani-Hamed, S. Dimopoulos, G. Dvali, Phys. Rev. D 59, 086004 (1999)

3. I. Antoniadis, N. Arkani-Hamed, S. Dimopoulos, G. Dvali, Phys. Lett. B 436, 257 (1998)

4. P. Horava, E. Witten, Nucl. Phys B475, 94 (1996)

5. P. Horava, E. Witten, Nucl. Phys. B460, 506 (1996)

6. L. Randall, R. Sundrum, Phys. Rev. Lett. 83, 3370 (1999)

7. N. Kaloper, Phys. Rev. D 60, 123506 (1999)

8. T. Nihei, Phys. Lett. B 465, 81 (1999)

9. H.B. Kim, H.D. Kim, Phys. Rev. D 61, 064003 (2000)

10. A.G. Cohen, D.B. Kaplan, Phys. Lett. B 470, 52 (1999)

11. C.P. Burgess, L.E. Ibanez, F. Quevedo, ibid 447, 257 (1999)

12. A. Chodos, E. Poppitz, ibid 471, 119 (1999)

13. T. Gherghetta, M. Shaposhnikov, Phys. Rev. Lett. 85, 240 (2000)

14. G.F. Giudice, R. Rattazzi, J.D. Wells, Nucl. Phys. B 544, 3 (1999)

15. T. Paul, S. SenGupta, Phys. Rev. D 95(11), 115011 (2017)

16. R. Marteens, K. Koyama, Brane-World Gravity. Living Rev. Rel. 13, $5(2010)$

17. A. Das, D. Maity, T. Paul, S. SenGupta, Eur. Phys. J. C 77(12), 813 (2017)

18. S. Kanno, J. Soda, Phys. Rev. D 66, 083506 (2002)

19. T. Shiromizu, K. Maeda, M. Sasaki, Phys. Rev. D 62, 024012 (2000) 
20. S. Chakraborty, S. SenGupta, Eur. Phys. J. C 75(11), 538 (2015)

21. W.D. Goldberger, M.B. Wise, Phys. Rev. Lett. 83, 4922 (1999)

22. W.D. Goldberger, M.B. Wise, Phys. Lett B 475, 275-279 (2000)

23. C. Csaki, M.L. Graesser, D. Graham, Kribs. Phys. Rev. D. 63, $065002(2001)$

24. J. Lesgourgues, L. Sorbo, Goldberger-Wise variations. Phys. Rev. D 69, 084010 (2004)

25. S. Das, D. Maity, S. SenGupta, J. High Energy Phys. 05, 042 (2008)

26. S. Anand, D. Choudhury, Anjan A. Sen, S. SenGupta, Phys. Rev. D 92(2), 026008 (2015)

27. A. Das, H. Mukherjee, T. Paul, S. SenGupta, Eur. Phys. J. C 78(2), 108 (2018)

28. T. Paul, S. SenGupta, Phys. Rev. D 93(8), 085035 (2016)

29. S. Chakraborty, S. SenGupta, Eur. Phys. J. C 74(9), 3045 (2014)

30. S. Kumar, A.A. Sen, S. SenGupta, Phys. Lett. B 747, 351-356 (2015)

31. S. Nojiri, S.D. Odintsov, Physics Reports 505, 59144 (2011)

32. S. Nojiri, S.D. Odintsov, V.K. Oikonomou, Phys. Rept. 692, 1-104 (2017)

33. S. Capozziello, S. Nojiri, S.D. Odintsov, Phys. Lett. B 781, 99-106 (2018)

34. R.G. Cai, L.M. Cao, Y.P. Hu, N. Ohta, Phys. Rev. D 80, 104016 (2009)

35. S.M. Carroll, A. De, V. Felice, D.A. Duvvuri, M. Easson, M.S.Turner Trodden, Phys. Rev. D 71, 063513 (2005)

36. F. Schmidt, M. Liguori, S. Dodelson, Phys. Rev. D 76, 083518 (2007)

37. S. Nojiri, S.D. Odintsov, V.K. Oikonomou, Phys. Lett. B 775, 4449 (2017)

38. V. Faraoni, Phys. Rev. D 75, 067302 (2007)
39. K. Bamba, C.Q. Geng, S. Nojiri, S.D. Odintsov, Europhys. Lett. 89, 50003 (2010)

40. T.P. Sotiriou, V. Faraoni, S. Liberati, Int. J. Mod. Phys. D 17, 399 (2008)

41. T.P. Sotiriou, V. Faraoni, Rev.Mod.Phys 82, 451497 (2010)

42. A. De Felice, S. Tsujikawa, Living Rev. Rel. 13, 3 (2010)

43. A. Paliathanasis, Class. Quant. Grav.33no 7, 075012 (2016)

44. S. Nojiri, S. D. Odintsov, Phys.Lett.B 631 (2005)

45. N. Banerjee, T. Paul, Eur. Phys. J. C 78(2), 130 (2018)

46. G. Cognola, E. Elizalde, S. Nojiri, S.D. Odintsov, S. Zerbini, Phys. Rev. D 73, 084007 (2006)

47. J.D. Barrow, S. Cotsakis, Phys. Lett. B 214, 515-518 (1988)

48. S. Capozziello, R. de Ritis, A. A. Marino, Class. Quant. Grav.14, 32433258 (1997)

49. S. Bahamonde, S. D. Odintsov, V. K. Oikonomou, M. Wright, arXiv: 1603.05113 [gr-qc]

50. R. Catena, M. Pietroni, L. Scarabello, Phys. Rev. D 76, 084039 (2007)

51. N. Banerjee, T. Paul, Eur. Phys. J. C 77(10), 672 (2017)

52. S. SenGupta, S. Chakraborty, Eur. Phys. J. C 76(10), 552 (2016)

53. S. Chakraborty, S. SenGupta, Phys. Rev. D 90(4), 047901 (2014)

54. S.B. Giddings, Phys. Rev. D 68, 026006 (2003)

55. J.J. Blanco-Pilado, D. Schwartz-Perlov, A. Vilenkin, JCAP 12, 006 (2009)

56. S.R. Coleman, Phys. Rev. D 152929 (1977) [Erratum ibid. D 16 (1977) 1248] [SPIRES];

57. C.G. Callan, Jr, S.R. Coleman, Phys. Rev. D 161762 [SPIRES] (1977)

58. S.R. Coleman, F. De Luccia, Phys. Rev. D 21, 3305 (1980)

59. S.J. Parke, Phys. Lett. B 121313 [SPIRES] (1983) 